\title{
25 Research Square \\ Incubation Period of COVID-19 From 11545 Patients in Observation Study
}

\section{Cheng Cheng}

Zhengzhou University

Dongdong Zhang

Zhengzhou University

\section{Dejian Dang}

The Fifth Affiliated Hospital of Zhengzhou University

Juan Geng

Zhengzhou University of Light Industry

\section{Peiyu Zhu}

Zhengzhou University

Mingzhu Yuan

Zhengzhou University

\section{Ruonan Liang}

Zhengzhou University

Haiyan Yang

Zhengzhou University

\section{Yuefei Jin}

Zhengzhou University

Jing Xie

Peter MacCallum Cancer Centre

Shuaiyin Chen ( $\nabla$ sychen@zzu.edu.cn )

Zhengzhou University

\section{Guangcai Duan}

Zhengzhou University

\section{Research Article}

Keywords: COVID-19, Incubation period, Mainland China

Posted Date: April 5th, 2021

DOI: https://doi.org/10.21203/rs.3.rs-332666/v1 
License: (c) (i) This work is licensed under a Creative Commons Attribution 4.0 International License. Read Full License 


\section{Abstract \\ Background}

The incubation period is a key index of epidemiology in understanding of the spread of infectious diseases and the decision-making of the disease control. However, the incubation period of the emerging COVID-19 is still unclear.

\section{Methods}

Between January 19, 2020 and September 21, 2020, we collected information on 11545 patients in Mainland China outside Hubei. The 218 patients with precise data was validation population. The incubation period was fitted with lognormal model by the coarseDataTools package in R.

\section{Results}

In 11545 patients, the mean incubation period of COVID-19 was 7.1 days ( $95 \%$ Confidence interval [CI], 7.0-7.2). About $5.4 \%$ of patients had precise incubation period less than 3 days, $10.2 \%$ longer than 14 days, and $2.1 \%$ longer than 21 days. There was no statistically significant difference in incubation period between male and female $(P=0.603)$. It was similar in the 218 patients. The mean accurate incubation period was 6.8 days (6.2-7.4). Of which, $14.7 \%(32 / 218)$ of patients had incubation period less than 3 days, $12.4 \%(27 / 218)$ longer than 14 days, and $0.9 \%(2 / 218)$ longer than 21 days.

\section{Conclusions}

For COVID-19, the mean incubation period is 7.1 days and $10.2 \%$ of patients developed disease 14 days after infection, which challenges the current 14-day quarantine strategy.

\section{Background}

Coronavirus disease 2019 (COVID-19), caused by severe acute respiratory syndrome coronavirus (SARSCoV-2), has spread over 223 countries and was declared a pandemic on March 11, 2020, by World Health Organization ${ }^{1}$. The number of COVID-19 patients is rapidly increasing globally. There were more than 113.5 million confirmed cases and 2.5 million deaths globally by March 2, 2021 ${ }^{1}$. The pandemic of COVID-19 has become a worldwide public health issue.

Incubation period, known as the interval between initial infection and onset of disease, is an important index to characterize the spread of infectious disease and formulate quarantine measures. For example, the mean incubation period is often used to calculate the reproduction number, and the maximum incubation period is the evidence to inform the duration of quarantine. In previously published studies, the 
mean/median incubations period of COVID-19 varied from 2.87 days $^{2}$ to 17.6 days $^{3}$. Most patients with COVID-19 had exposure intervals longer than one day, so the exact date of their infection cannot be easily determined. Several studies used the earliest exposure time to define of the infection date and provided mean incubation periods of COVID-19 of about 8 days in mainland China outside Hubei province ${ }^{4-6}$. Other studies used the latest exposure time to define of the date of infection and reported a shorter mean incubation periods of COVID-19 of about 5 days ${ }^{7-9}$. Obviously, the incubation period of COVID-19 was overestimated using the earliest exposure and underestimated using the latest exposure, which resulted in unreliable incubation periods. For the patients with unclear data on the date of infection or the date of onset of disease, Nicholas et al provided a method to represent their data as doubly interval-censored to accurate the incubation period distribution ${ }^{10}$. This method has been used to estimate the incubation period in several previously studies with less than 1200 patients with COVID-19 ${ }^{11-13}$, but the sample size of these studies is much smaller than the total number of patients. Therefore, a study with large sample is needed to estimate the incubation period of COVID-19.

This study aims to estimate the incubation period of COVID-19. We collect the information on confirmed cases with COVID-19 in mainland China outside Hubei and extract data from 11,545 patients to estimate the incubation periods. To verify the reliability of incubation period, sensitivity analysis is performed in patients with precise date of infection and onset of disease.

\section{Patients And Methods}

\section{Study Population}

By September 21, 2020, a total of 17168 patients with COVID-19 have been diagnosed with positive nucleic acid and clinical symptoms in Mainland China outside Hubei. Among them, the available information of 11545 patients can be collected from the national and local health commissions in China 14. We extracted the patients' information including age, sex, date of the earliest exposure, date of the latest exposure, date of the nucleic acid test report, date of onset of disease, date of first visit, and date of diagnosis. All 11545 patients were included to estimate the incubation period.

Further, 218 patients with precise date of infection and onset of disease were selected to estimate the accurate incubation period. The exclude criteria included that the patient 1) did not report the date of onset of disease, 2) did not report the date of the earliest exposure or the date of the latest exposure, and 3) had exposure intervals longer than 1 day.

This study was approved by the Zhengzhou University Medical Ethics Committee (Zhengzhou, China). The information on all cases was collected from publicly available sources, and informed consent was waived.

\section{Date Of Onset And Date Of Infection}


The incubation period was defined as the time interval between the date of infection and the date of onset of disease. There were 7303 patients with the date of onset of disease (Definition 1). In the patients without the date of onset, we defined the date of onset as the date of first visit minus 2.5 days (Definition 2) or the date of diagnosis minus 5.4 days (Definition 3) ${ }^{14}$. The date of infection is between the date of the earliest exposure and the date of the latest exposure. If exposed to the source of infection only within one day, the date of infection is the date of exposure, otherwise it was represented as doubly intervalcensored ${ }^{10}$. When the date of the latest exposure was not reported, we defined it as the earliest date among date of the nucleic acid positive, date of onset of disease, date of first visit, and date of diagnosis minus one days. When the date of the earliest exposure was not reported or the exposure interval (from the earliest exposure to the latest exposure) was longer than 14 days, we defined it as the date of the latest exposure minus 14 days.

\section{Statistical Analysis}

We presented mean ((95\% Confidence interval [CI]) for continuous variables and count (percentage) for categorical variables. The distribution curve of the incubation period was fitted with lognormal model by "coarseDataTools" package in the R software ${ }^{10}$. Wilcoxon signed-rank test was used to compare differences in subgroup analysis by age and gender. Significantly difference was identified with adjusted $P$ value with false discovery rate at 0.05 . Sensitivity analysis explored the impact of different definitions of date of onset.

Statistical analyses were conducted using $R$ version 4.0.2. Two-sided $P<0.05$ indicated statistically significant.

\section{Results}

\section{Estimating incubation period in observation study}

Table 1 presents the characteristics of 11545 patients with COVID-19. The median age was 45 years (IQR: $33-56)$, and there were 5814 males (52.7\%). The mean incubation period is 7.1 days $(95 \% \mathrm{Cl}, 7.0-7.2)$ in total population. The fitted curve (Fig. 1) shows that there are $5.4 \%$ of patients with the incubation period less than 3 days, $10.2 \%$ more than 14 days, and $2.1 \%$ more than 21 days. 
Table 1

Characteristics of 11545 patients with Coronavirus disease 2019

\begin{tabular}{|llll|}
\hline & $\begin{array}{l}\text { No. of patients } \\
\text { (\%) }\end{array}$ & $\begin{array}{l}\text { Mean incubation period } \\
(95 \% \mathrm{Cl})\end{array}$ & $P$ \\
\hline Total & $11545(100 \%)$ & $7.1(7.0-7.2)$ & 0.603 \\
\hline Gender & & & \\
\hline Male & $5814(52.7 \%)$ & $7.2(7.0-7.4)$ & $<0.001$ \\
\hline Female & $5215(47.3 \%)$ & $7.1(6.9-7.3)$ & \\
\hline Age, years & & & \\
\hline$<18$ & $460(4.4 \%)$ & $8.6(8.0-9.3)$ & \\
\hline $18-40$ & $3789(36.3 \%)$ & $7.2(7.0-7.4)$ & \\
\hline $41-60$ & $4425(42.3 \%)$ & $7.1(6.9-7.2)$ & \\
\hline$>60$ & $1777(17.0 \%)$ & $7.0(6.6-7.3)$ & \\
\hline Definition of date of onset & & & \\
\hline Definition 1 & $7303(63.2 \%)$ & $7.1(7.0-7.3)$ & \\
\hline Definition 2 & $2168(18.8 \%)$ & $7.0(6.7-7.3)$ & \\
\hline Definition 3 & $2074(18.0 \%)$ & $7.3(7.0-7.6)$ & \\
\hline Cl, Confidence interval & & & \\
\hline
\end{tabular}

Definition 1, using the reported date of onset;

Definition 2, defined as the date of first visit minus 2.5 days;

Definition 3, defined as the date of diagnosis minus 5.4 days.

The population-specific incubation periods are presented in Table 1 and Fig. 2. The mean incubation period is 7.2 days $(7.0-7.4)$ in male and 7.1 days $(95 \% \mathrm{Cl}, 6.9-7.3)$ in female $(P=0.603)$. Moreover, it is 8.6 days $(95 \% \mathrm{Cl}, 8.0-9.3), 7.2$ days $(95 \% \mathrm{Cl}, 7.0-7.4), 7.1$ days $(95 \% \mathrm{Cl}, 6.9-7.2)$, and 7.0 days $(95 \% \mathrm{Cl}$, 6.6-7.3) in patients under 18 years, $18-40$ years, $41-60$ years, and over 60 years, respectively $(P<$ 0.001). The incubation period is robust among Definition 1 (mean [95\% $\mathrm{Cl}] 7.1$ days [7.0-7.3], Definition 2 (7.0 days $[6.7-7.3])$, and Definition 3 (7.3 days $[7.0-7.6])(P=0.319)$.

Table 2 presents the characteristics of 218 patients with precise data. The median age was 47 years (IQR: $35-56)$, and there were 121 males (56.3\%). The mean accurate incubation period is 6.8 days $(95 \% \mathrm{Cl}$, $6.2-7.4)$. Figure 3 shows the distribution of the accurate incubation periods in 218 patients. The shortest 
and longest accurate incubation period are 1 day and 26 days, respectively. There are 8 patients $(3.7 \%)$ with an incubation period of 1 day, 32 (14.7\%) not exceeding 3 days, $27(12.4 \%)$ more than 14 days, and 2 $(0.9 \%)$ more than 21 days.

Table 2

Characteristics of 218 patients with Coronavirus disease 2019

\begin{tabular}{|llll|}
\hline & $\begin{array}{l}\text { No. of patients } \\
(\%)\end{array}$ & $\begin{array}{l}\text { Mean incubation period } \\
(95 \% \mathrm{Cl})\end{array}$ & $\boldsymbol{P}$ \\
\hline Total & $218(100 \%)$ & $6.8(6.2-7.4)$ & 0.145 \\
\hline Gender & & & \\
\hline Male & $121(56.3)$ & $6.3(5.4-7.2)$ & 0.009 \\
\hline Female & $94(43.7)$ & $7.5(6.7-8.4)$ & \\
\hline Age, years & & & \\
\hline$<18$ & $6(2.8)$ & $9.3(5.3-16.3)$ & \\
\hline $18-40$ & $73(34.1)$ & $7.7(6.6-9.0)$ & \\
\hline $41-60$ & $98(45.8)$ & $5.7(4.9-6.6)$ & \\
\hline$>60$ & $37(17.3)$ & $8.0(6.5-9.8)$ & \\
\hline Cl, Confidence interval & & \\
\hline
\end{tabular}

The population-specific distributions of the accurate incubation periods are shown in Table 2 and Fig. 4. The mean incubation period is 9.3 days $(95 \% \mathrm{Cl}, 5.3-16.3), 7.7$ days $(95 \% \mathrm{Cl}, 6.6-9.0), 5.7$ days $(95 \% \mathrm{Cl}$, $4.9-6.6)$, and 8.0 days $(95 \% \mathrm{Cl}, 6.5-9.8)$ in patients under 18 years, $18-40$ years, $41-60$ years, and over 60 years, respectively ( $41-60$ years vs. $18-40$ years: $P=0.028 ; 41-60$ years vs. $\geq 61$ years: $P=0.041$ ). The mean incubation period is 6.3 days $(95 \% \mathrm{Cl}, 5.4-7.2)$ in male and 7.5 days $(95 \% \mathrm{Cl}, 6.7-8.4)$ in female $(P=0.145)$.

\section{Discussion}

This study has the largest sample to estimate the incubation period of COVID-19. For 11545 patients, the mean incubation period is 7.1 days, $10.2 \%$ of patients with incubation period more than 14 days, and $2.1 \%$ more than 21 days. Moreover, the incubation period of COVID-19 is associated with age. The similar evidences are presented in 218 patients with the accurate incubation period.

The mean incubation period of COVID-19 is about seven days. In the large COVID-19 patients' population, we report a mean incubation period of 7.1 days $(95 \% \mathrm{Cl}, 7.0-7.2)$. Similarly, the mean accurate incubation period was 6.8 days $(95 \% \mathrm{Cl}, 6.2-7.4)$ in 218 patients, supporting that the finding is reliability in large population. Lu et al reported a median incubation period of 7.2 days $(95 \% \mathrm{Cl}, 6.9-7.5)$ in 1158 patients with COVID-19 based on the Weibull distribution, which also used the interval-censored data ${ }^{13}$. 
Their result is consistent with ours, but the $95 \% \mathrm{Cl}$ of median incubation period is wider. However, the studies using the earliest exposure date had the longer median incubation period than ours, which showed the median incubation period of about 8 days ${ }^{5} 8$. Conversely, a study using the last exposure date reported a median incubation period of 5 days, which shorted than ours ${ }^{7}$. Similarly, two other studies using last exposure date reported short median incubation periods of 3 days and 4.8 days, respectively ${ }^{15} 16$. Obviously, these biases are mainly due to the inaccurate date of infection. The incubation period was overestimated using the date of the earliest exposure and underestimated using the date of the last exposure date. We conducted a meta-analysis which included 27 studies and presented mean incubation period as 6.3 days (95\% Cl: $5.7-7.0)$ in mainland China (data were shown in Supplementary File). It also consistent with the results of our observation study Therefore, our study provided a reliable incubation period of COVID-19, which is a robust evidence for understanding SARSCov-2 transmission.

The 14 days quarantine strategy is challenged. In our study, about $10.2 \%$ patients developed disease 14 days after infection, and $2.1 \%$ developed disease 21 days after infection. A study focused on the importation risk of COVID-19 is consistent with our findings, which reported that $9 \%$ of patients had a negative report by nucleic acid test during the 14 th day of isolation ${ }^{17}$. Therefore, we speculate that, if only relying on clinical symptoms or nucleic acid test results, the 14-day isolation strategy will result in about $10 \%$ of patients not being recognized as confirmed cases. When a stricter strategy is implemented, more patients will be identified. In Wuhan city, after strict isolation of all residents on January 23, the number of new cases with COVID-19 decreased rapidly, with a $50 \%$ reduction after 14 days (February 6 ) and a $75 \%$ reduction after 28 days (February $20{ }^{18}$. As we know, very strict isolation will bring a huge social burden. Should all regions adopt a Wuhan-style quarantine strategy to prevent spread of SARSCoV-2? Our findings suggest that the people should be quarantined for 21 days or more who had a high risk of being infected with SARS-Cov-2, such as contacting with the patients infected with SARS-CoV-2 or coming from cities or country where COVID-19 is in epidemic.

Additionally, the potential patients should be isolated as soon as possible. We found that about $15 \%$ of patients had an incubation period less than 3 days. In India, there were $25 \%$ of patients had an incubation period less than 3 days (25th percentile: 3.0 days) ${ }^{19}$. The one third of patients in Singapore had an incubation period of less than or equal to 3 days ${ }^{20}$. The previous studies report that patients with COVID19 are infectious before they develop symptoms ${ }^{21}$. Our study found that half patients develop symptoms within 7 days after infection. A meta-analysis showed that the mean serial interval of COVID-19 was 5.5 days ${ }^{22}$. So that, for COVID-19, the serial interval is short than incubation period. We speculate that the spread of SARS-CoV-2 occurs on average 1.5 days before the onset of disease. If all close contacts are quarantined at the third day after infection, more than $15 \%$ of the people infected with SARS-Cov- 2 in close contacts may have infected others. When COVID-19 patients and their close and sub-close contacts are quarantined on the first time, the further transmission will be terminated. 
The incubation period of COVID-19 varies by age. We found that the incubation period is related with age. In 218 patients, the incubation period presented a U-shaped curve with increasing age. The middle-aged group (41-60 years) had the shortest incubation period than other groups, especially the elderly group ( $\geq$ 61 years) and 18-40 years group. The previous study with 136 patients had reported a similar agespecific distribution of incubation period with, the shortest incubation period in patients aged from 45 to 59 years ${ }^{6}$. Another study with 2555 patients also found a U-shaped curve distribution of incubation period in patients ${ }^{5}$. The mechanism of the effect of age on COVID-19 incubation period is unclear. Possible explanations include a less intense immune response, leading to a delay in the onset of symptoms and shorter exposure time and exposure rate in the elderly and children.

However, the incubation period has no difference between male and female. Previous study suggests that male is more susceptible to COVID-19, which may be due to the higher plasma concentration of ACE2 ${ }^{23}$. However, our study did not observe a difference in incubation period by gender. Nie et al also showed an insignificant difference in incubation period between male and female ( 5 days vs. 4 days; $P=0.22)^{7}$. Yang et al presented the similar conclusion ${ }^{24}$. The evidence suggests that gender maybe not a factor in the incubation period of COVID-19.

Several limitations need to be stated in the present study. First. the selection bias may also exist. although the sample size in this study is larger, there was 5623 patients with missing information. Moreover, it only accounts for a small part of the total number of cases in Mainland China outside to estimate the accurate incubation period. Second, there may be recall bias that the accuracy of the selfreported date of onset by patients may be affected. Third, although there is no significant difference in incubation periods between different definitions of the date of onset, the estimated date of onset may be biased from the actual date of onset. Finally, the date of infection in most cases is unclear, which affects the accuracy of incubation period.

\section{Conclusions}

This study provides the reliable incubation periods of COVID-19, which helps to understand the transmission of disease and formulate prevention measures. The mean incubation period is about 7 days in larger population or in patients with precise data. Moreover, $5.4 \%$ of patients had incubation period within 3 days, only $90 \%$ within 14 days, but $98 \%$ within 21 days. Therefore, the 14 -day quarantine period may not be enough, but 21 days or even stricter quarantine measures may be effective to control the spread of SARS-CoV-2. It is also necessary to isolate people exposure to COVID-19 cases as soon as possible.

\section{List Of Abbreviations}

ACE2

angiotensin converting enzyme 2; Cl:Confidence interval; COVID-19:Coronavirus disease 2019; IQR:interquartile range; 2019-nCoV:novel coronavirus 2019; NCP:novel coronavirus pneumonia; 
SD:standard deviation; SARS-CoV-2:severe acute respiratory syndrome coronavirus.

\section{Declarations}

\section{Ethical Approval and Consent to participate}

This study was approved by the Zhengzhou University Medical Ethics Committee (Zhengzhou, China). The information on all cases was collected from publicly available sources, and Consent to participate was waived.

\section{Consent for publication}

Not applicable.

\section{Availability of data and materials}

The data used for this article is available from the corresponding author on reasonable request..

\section{Competing interests}

The authors declare that they have no competing interests.

\section{Fundings}

The work was funded by the National Natural Science Foundation of China (N0.82073618 and NO.82002147); the Key Scientific Research Projects in Colleges and Universities of Henan Province [20A330004]; the National Science and Technology Specific Projects (NO.2018ZX10301407)

\section{Authors' contributions}

Shuaiyin Chen and Cheng Cheng designed and implemented this research; Cheng Cheng wrote the manuscript; Juan Geng, Peiyu Zhu, Mingzhu Yuan, and Ruonan Liang collected and screened individual information on COVID-19 patients; Chengcheng, Dongdong Zhang, and Dejian Dang analyzed the data; Jing Xie made some suggestions and polished the language; Shuaiyin Chen provided suggestions for the revision of the paper; Guangcai Duan initiated this project, interpreted the results, and revised the manuscript. All authors reviewed the manuscript and approved this version to be published.

\section{Acknowledgments}

The authors would like to thank everyone who provided support for this study, especially thank Professor Junyuan Yang, Professor Xichao Duan, and Professor Xuezhi Li.

\section{Authors' information}


1 Department of Epidemiology and Health Statistics, College of Public Health, Zhengzhou University, No. 100 Kexue Avenue, Zhengzhou, Henan 450001, People's Republic of China. ${ }^{2}$ Department of Nutrition and Food Hygiene, College of Public Health, Zhengzhou University, No. 100 Kexue Avenue, Zhengzhou, Henan 450001, People's Republic of China. . ${ }^{3}$ Infection Prevention and Control Department, The Fifth Affiliated Hospital of Zhengzhou University, No.3 Kangfuqian Street, Zhengzhou, Henan 450052, People's Republic of China. ${ }^{4}$ Henan Key Laboratory of Molecular Medicine, Zhengzhou University, No. 100 Kexue Avenue, Zhengzhou, Henan 450001, People's Republic of China. ${ }^{5}$ Centre for Biostatistics and Clinical Trials (BaCT), Peter MacCallum Cancer Centre, No. 305 Grattan Street, Victoria, 3000, Australia.

\section{References}

1. World Health Organization. COVID-19 Weekly Epidemiological Update (2 March 2021).

https://www.who.int/publications/m/item/weekly-epidemiological-update-2-march-2021. Accessed March 42021

2. Chun JY, Baek G, Kim Y. Transmission onset distribution of COVID-19. Int J Infect Dis. Oct 2020;99:403-407. doi:10.1016/j.ijid.2020.07.075

3. Xie S, Zhang G, Yu H, et al. The epidemiologic and clinical features of suspected and confirmed cases of imported 2019 novel coronavirus pneumonia in north Shanghai, China. Ann Transl Med. May 2020;8:637. doi:10.21037/atm-20-2119

4. You C, Deng Y, Hu W, et al. Estimation of the time-varying reproduction number of COVID-19 outbreak in China. Int J Hyg Environ Health. Jul 2020;228:113555. doi:10.1016/j.ijheh.2020.113555

5. Xiao Z, Xie X, Guo W, et al. Examining the incubation period distributions of COVID-19 on Chinese patients with different travel histories. J Infect Dev Ctries. Apr 30 2020;14:323-327. doi:10.3855/jidc. 12718

6. Kong TK. Longer incubation period of coronavirus disease 2019 (COVID-19) in older adults. Aging Med (Milton). Jun 2020;3:102-109. doi:10.1002/agm2.12114

7. Nie X, Fan L, Mu G, et al. Epidemiological Characteristics and Incubation Period of 7015 Confirmed Cases With Coronavirus Disease 2019 Outside Hubei Province in China. J Infect Dis. Jun 16 2020;222:26-33. doi:10.1093/infdis/jiaa211

8. Qin J, You C, Lin Q, et al. Estimation of incubation period distribution of COVID-19 using disease onset forward time: A novel cross-sectional and forward follow-up study. Sci Adv. Aug 2020;6:eabc1202. doi:10.1126/sciadv.abc1202

9. Ren X, Li Y, Yang X, et al. Evidence for pre-symptomatic transmission of coronavirus disease 2019 (COVID-19) in China. Influenza Other Respir Viruses. Jan 2021;15:19-26. doi:10.1111/irv.12787

10. Reich NG, Lessler J, Cummings DA, et al. Estimating incubation period distributions with coarse data. Stat Med. Sep 30 2009;28:2769-84. doi:10.1002/sim.3659

11. Wu J, Huang Y, Tu C, et al. Household Transmission of SARS-CoV-2, Zhuhai, China, 2020. Clin Infect Dis. Nov 19 2020;71:2099-2108. doi:10.1093/cid/ciaa557 
12. Lauer SA, Grantz KH, Bi Q, et al. The Incubation Period of Coronavirus Disease 2019 (COVID-19) From Publicly Reported Confirmed Cases: Estimation and Application. Annals of internal medicine. Mar 10 2020;doi:10.7326/m20-0504

13. Lu QB, Zhang Y, Liu MJ, et al. Epidemiological parameters of COVID-19 and its implication for infectivity among patients in China, 1 January to 11 February 2020. Euro Surveill. Oct 2020;25. doi:10.2807/1560-7917.ES.2020.25.40.2000250

14. Zhang J, Litvinova M, Wang W, et al. Evolving epidemiology and transmission dynamics of coronavirus disease 2019 outside Hubei province, China: a descriptive and modelling study. The Lancet Infectious Diseases. 2020;20:793-802. doi:10.1016/s1473-3099(20)30230-9

15. Yang N, Shen Y, Shi C, et al. In-flight transmission cluster of COVID-19: a retrospective case series. Infect Dis (Lond). Nov - Dec 2020;52:891-901. doi:10.1080/23744235.2020.1800814

16. Shi $P$, Gao Y, Shen Y, et al. Characteristics and evaluation of the effectiveness of monitoring and control measures for the first 69 Patients with COVID-19 from 18 January 2020 to 2 March in Wuxi, China. Sustain Cities Soc. Jan 2021;64:102559. doi:10.1016/j.scs.2020.102559

17. Dickens BL, Koo JR, Lim JT, et al. Strategies at points of entry to reduce importation risk of COVID-19 cases and reopen travel. Journal of travel medicine. Dec 23 2020;27.doi:10.1093/jtm/taaa141

18. Pan A, Liu L, Wang C, et al. Association of Public Health Interventions With the Epidemiology of the COVID-19 Outbreak in Wuhan, China. JAMA. May 19 2020;323:1915-1923. doi:10.1001/jama.2020.6130

19. Patrikar SR, Kotwal A, Bhatti VK, et al. Incubation Period and Reproduction Number for Novel Coronavirus 2019 (COVID-19) Infections in India. Asia Pac J Public Health. Nov 2020;32:458-460. doi:10.1177/1010539520956427

20. Pung R, Chiew CJ, Young BE, et al. Investigation of three clusters of COVID-19 in Singapore: implications for surveillance and response measures. Lancet (London, England). Mar 28 2020;395:1039-1046. doi:10.1016/s0140-6736(20)30528-6

21. Guan Q, Liu M, Zhuang YJ, et al. [Epidemiological investigation of a family clustering of COVID-19]. Zhonghua liu xing bing xue za zhi = Zhonghua liuxingbingxue zazhi. Mar 9 2020;41:629-633. doi:10.3760/cma.j.cn112338-20200223-00152

22. Hussein M, Toraih E, Elshazli R, et al. Meta-Analysis on Serial Intervals and Reproductive Rates for SARS-CoV-2. Ann Surg. Nov 17 2020;doi:10.1097/SLA.0000000000004400

23. Sama IE, Ravera A, Santema BT, et al. Circulating plasma concentrations of angiotensin-converting enzyme 2 in men and women with heart failure and effects of renin-angiotensin-aldosterone inhibitors. Eur Heart J. May 14 2020;41:1810-1817. doi:10.1093/eurheartj/ehaa373

24. Yang L, Dai J, Zhao J, Wang Y, Deng P, Wang J. Estimation of incubation period and serial interval of COVID-19: analysis of 178 cases and 131 transmission chains in Hubei province, China. Epidemiol Infect. Jun 19 2020;148:e117. doi:10.1017/S0950268820001338

\section{Figures}




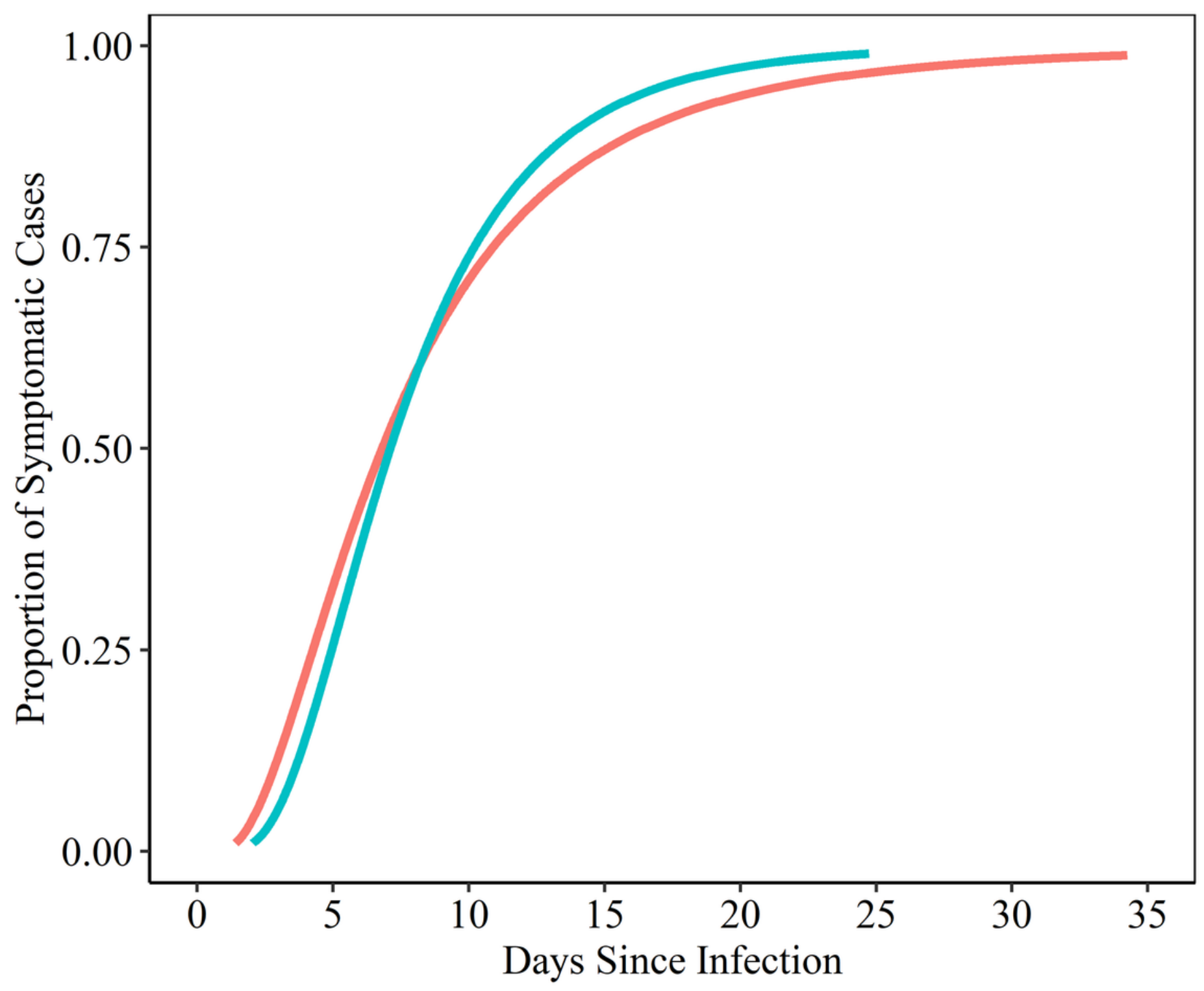

Figure 1

The fitted distribution curve of incubation period in 11545 patients with Coronavirus disease 2019 . 

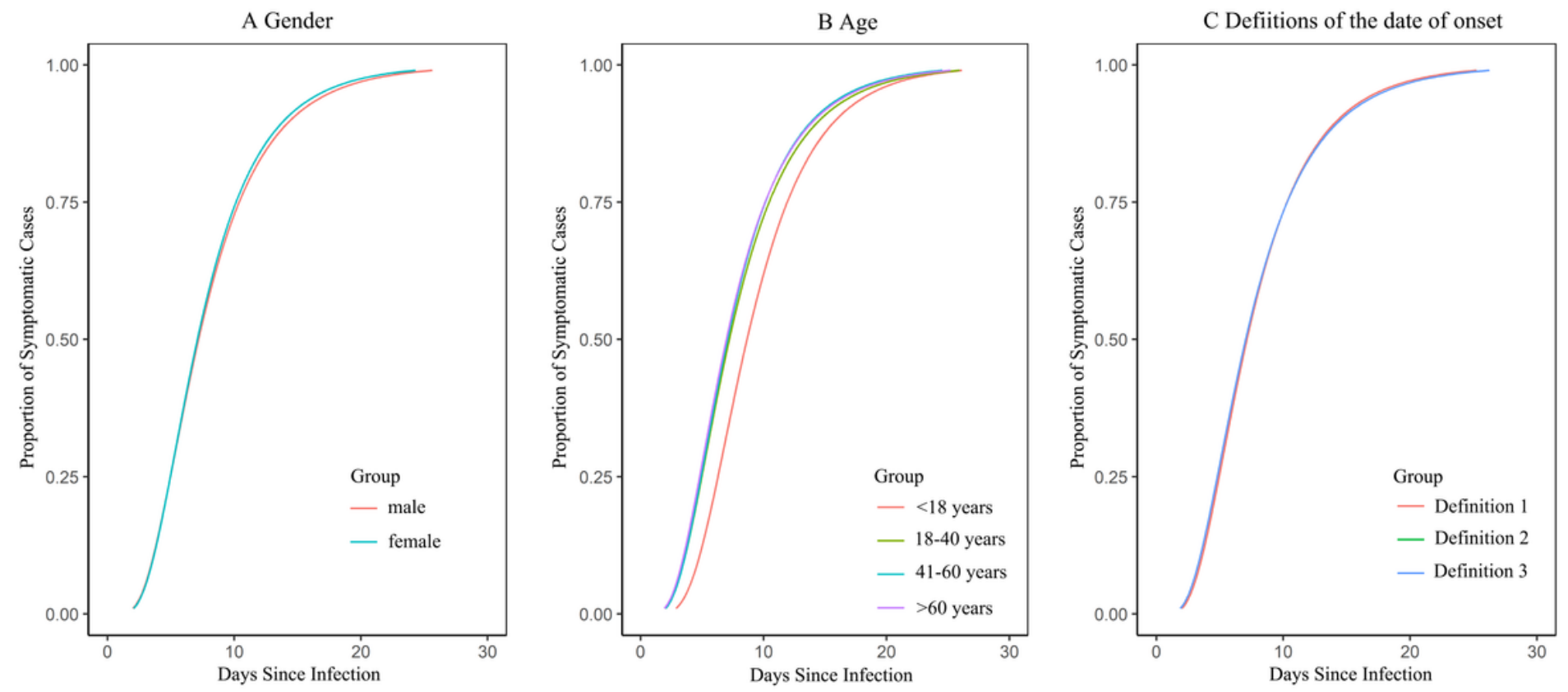

Figure 2

The population-specific fitted distribution curve of incubation period of Coronavirus disease 2019. A. by gender; B. by age; $\mathrm{C}$. by definition of the date of onset; and D, by the exposure interval. 


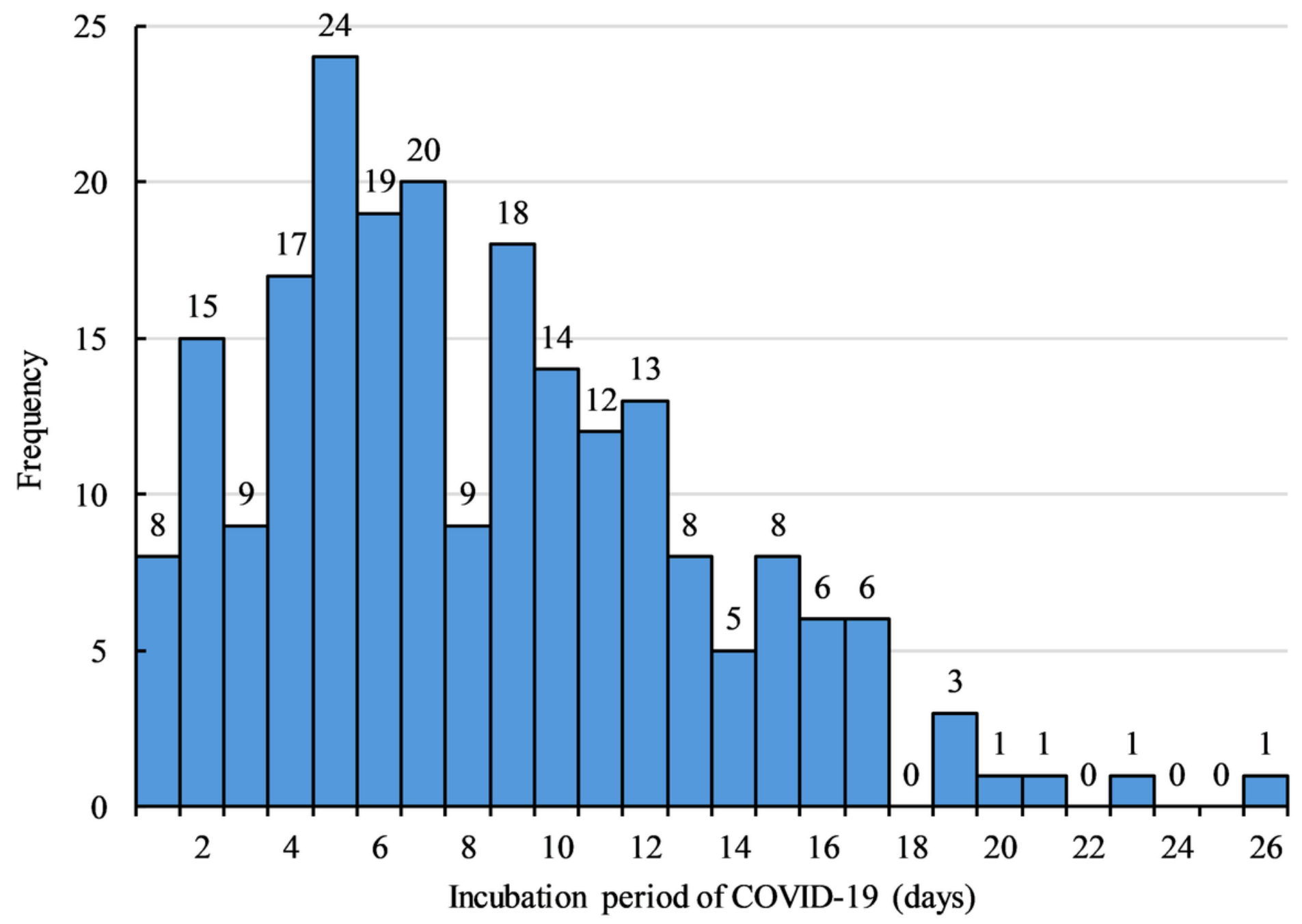

Figure 3

The distribution of individual incubation period of Coronavirus disease 2019 (COVID-19) for 218 patients. A. Histogram of incubation period. B. the fitted distribution curve of incubation period.
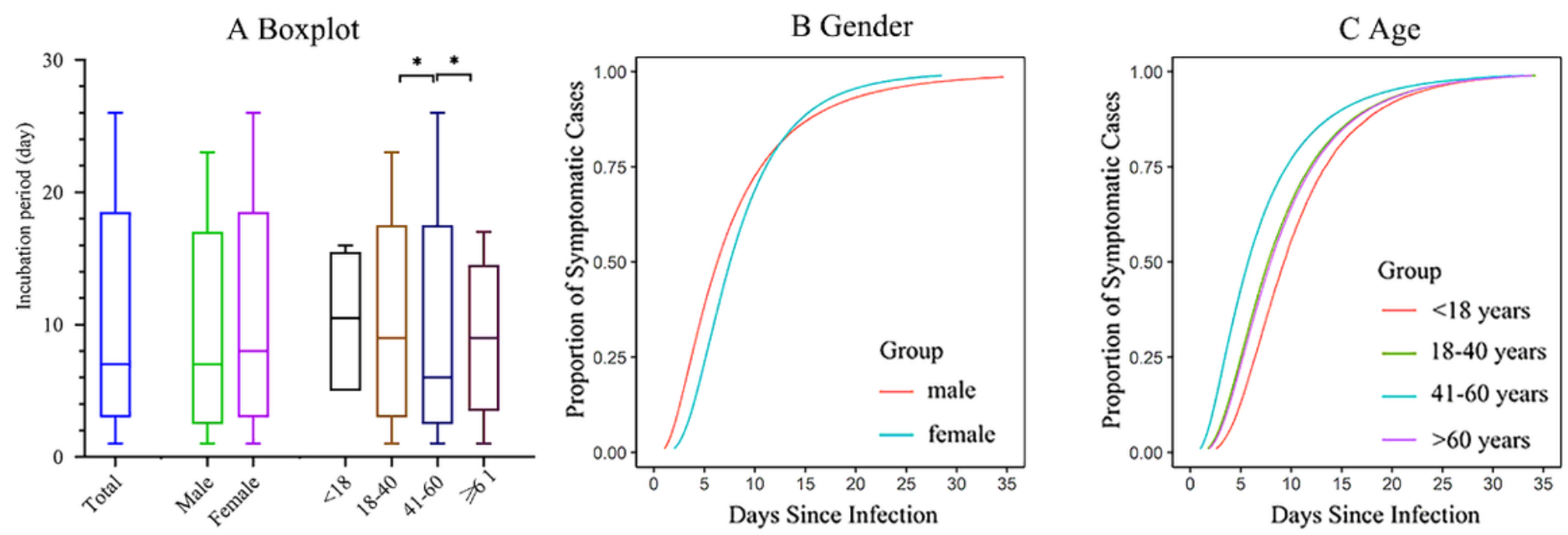

Figure 4 
The boxplot and population-specific fitted distribution curve of accurate incubation period of Coronavirus

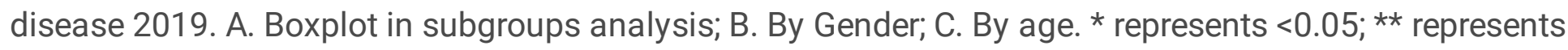
$<0.01$.

\section{Supplementary Files}

This is a list of supplementary files associated with this preprint. Click to download.

- Supplementarydata.docx 